\title{
Vector analysis of rotary motion perception
}

\author{
HANS WALLACH \\ Swarthmore College, Swarthmore, Pennsylvania \\ and \\ ANN O'LEARY \\ Stanford University, Stanford, California
}

\begin{abstract}
Five displays with light dots at two or three rim locations on a wheel that rolled inside a hoop of twice the wheel's diameter were observed. In this arrangement the motion paths of the rim dots are straight and are apparently more readily perceived than the cycloids on which rim dots move when the wheel rolls on a plane. Circular dot motion, the apparent result of vector analysis, is, however, often perceived as an alternative to linear dot motion. New findings that had been obtained in experiments with two of Johansson's motion patterns that demonstrated vector analysis were shown to apply to this rolling wheel display. They are here presented in the context of a recent interpretation of Johansson's findings. It regards the simultaneously perceived motions that Johansson's displays yield as the direct result of two kinds of proximal stimulation, made possible by vector analysis taking place between distal and proximal stimulation. The following findings are reported. Adding a stationary dot in the periphery of the displays increased linear and decreased circular perceived motion when the displays were freely viewed. The perception of linear motion, which is given by subject-relative stimulation, and the perception of circular motion, which is given as orientation change, can combine to form perceived elliptic motion paths. This happened frequently, regardless of whether the linear motion was given by means of ocular pursuit or as retinal image path.
\end{abstract}

A point on the edge of a rolling wheel moves through an arched path, called a cycloid. A cycloid is the kinematic resultant of two component motions, one circular and the other translatory. When a rolling wheel is observed under ordinary conditions, these two component motions are simultaneously perceived, and the cycloids are not seen. Only when, in the dark, a light dot is fixed to the wheel edge is its cycloid path seen. When a second light dot is added, the cycloid paths of the dots are no longer visible; rather, the two dots appear to move about each other. Experiments of this sort were first done by Duncker (1929), who credited Rubin (1927) for suggesting them. Duncker did not expose two points on the wheel edge but, in most of his experiments, a light dot at the wheel's center and another dot at a midpoint between the center and the wheel edge. Nevertheless, he often obtained reports that one dot circled about the other or that the dots revolved about a common center. Duncker thought of these motions as analogues to certain features of induced motion, to which he referred as "separation of systems."

Eventually, Johansson (1950) introduced the term "vector analysis" to refer to the perceptual process that takes

This work was supported by Grant 11089 from the National Institute of Mental Health to Swarthmore College, Hans Wallach, principal investigator. We are grateful to Martha Teghtsoonian for her help in writing this report and to Dennis R. Proffitt for his helpful criticism of our presentation.

H. Wallach's mailing address is: Department of Psychology, Swarthmore College, Swarthmore, PA 19081. place when rotational motions, instead of two or more cycloid motion paths, are perceived. The term, vector analysis, emphasizes the similarity with kinematics of events in which the cycloid motion paths are apparently broken down into different simultaneously perceived motions, a rotation and a translation. Moreover, Johansson invented a number of patterns of dot motions in which, as in the case of light dots on a rolling wheel, two kinematic components of the given motion paths were perceived instead of the given paths themselves. In the patterns he designed, the perceived "component" motions were both translatory.

Although Johansson assumed that this analysis into simultaneously perceived motions took place in perception, Wallach, Becklen, and Nitzberg (1985) have presented evidence to show that the simultaneously perceived motions result from simultaneously present stimulus conditions that evoke the different perceived motions. The different stimulations are provided by different kinematic components of the objective motion paths. In the physical world, two or more arbitrarily selected component motions, of which the objective motion is the resultant, are just as real as the actually present motion. Since stimuli are part of the physical world, stimulation will just as readily be produced by component motions as by the actually present motion. Whether the actually present motion or a pair of its components operate as stimuli depends on our perceptual equipment. Several stimuli that mediate motion perception represent different aspects of the 
objective motion pattern, and the simultaneously perceived motions are the outcome of the characteristics of the stimulation conditions involved. One of the stimuli that operate here is configurational change, which represents the components of the dot's motions that make up the changing figural relationships among them. Two other stimulus conditions, ocular pursuit and image displacement, represent the change in the location of a dot relative to the observer, its subject-relative displacement. Thus, no perceptual vector analysis needs to operate. Rather, the simultaneously perceived motions are the direct result of proximal stimulation.

One of the better known of Johansson's motion patterns may serve as an example: One dot moves up and down in reciprocating motion, and the other moves left and right. The two motions are in phase with each other and their paths cross at their centers. But these two motions are rarely perceived. Rather, the dots appear to move toward and past each other on oblique colinear paths. About two-thirds of the observers also report that the dots move together in the other oblique direction, perpendicular to the colinear paths.

Johansson (1950) ascribed the two simultaneous motions that each dot undergoes to an organizing activity of the nervous system, which, in the present instance, extracts the motion components the dots have in common, with the colinear paths of the dots being the residual motion components. Wallach (1965) proposed a minor change of Johansson's explanation, namely, that the colinear components of dot motion are extracted and the common motions of the dots are the residual components. Wallach et al. (1985) ascribed the simultaneously perceived motions to the multiple sensory responses to motion with which we are equipped. No extraction process need be postulated. Different conditions of stimulation that mediate motion perception represent different physical components of the objective motion patterns. Configurational change, which results from distance change between dots, represents the colinear components of the dots' motions. The subject-relative stimulus conditions represent either the motion components that the vertical and horizontal motions of the individual dots have in common when the dots form a group or the vertical and horizontal motions themselves. Why the latter motions are rarely reported will be discussed below.

Thus, Wallach et al.'s (1985) explanation of vector analysis is based on the rules of kinematics applied to physical motion patterns and on the existence of different stimulus conditions that operate in motion perception and fit different kinematic aspects of the physical motion pattern. Wallach et al. (1985) demonstrated the role of configurational change by adding stationary points in the surround of the horizontally and vertically moving dots. When these moving dots are given in a homogeneous field, the only configurational change that takes place is the distance change between them. It occurs along the line connecting them, and that determines the perceived motion resulting from configurational change. But when sta- tionary dots are added, the configurational changes between them and the moving dots represent the objective dot motions, which are then preponderantly perceived.

There is a difficulty with this explanation of vector analysis. When an object is given in the context of two stimulus conditions that cause it to move simultaneously in different directions, the two motion processes combine: a single path is perceived (see, e.g., Wallach, O'Leary, \& McMahon, 1982). If that were to happen in Johansson's motion patterns, it would supersede the result of vector analysis. Indeed, Wallach et al. (1985) found that although such a combining of the two motions did not occur always, it did occur frequently. When they experimented with Johansson's crossing-paths pattern just described, only 10 of 19 subjects reported the colinear paths previously reported. The other 9 subjects saw the paths of the dots cross under angles of $30^{\circ}$ to $40^{\circ}$. These paths were combinations of the colinear motions of the dot, given by configurational change $\left(0^{\circ}\right)$, and their horizontal and vertical motions, given by subject-relative stimulation $\left(90^{\circ}\right)$. This combining of the two motion processes apparently fails when the moving dots form a group. This prevents the dot motion from individually entering into process combination, and when that is avoided, the dots are seen to move in concert on parallel paths. This interpretation is supported by experiments Wallach et al. (1985) performed with another one of Johansson's motion patterns. It was found that the number of instances of combined motion was greatly diminished when group formation between the dots was strengthened.

As stated, the stimulus conditions that account for the apparent vector analysis in Johansson's pattern are the stimuli representing subject-relative displacement and configurational change. In rolling-wheel displays, orientation change-the change in the direction of the alignment between two dots-replaces configurational change. (We make a distinction between orientation change and configurational change, because they differ functionally. Orientation changes with respect to an absolute framework. Although the vertical and horizontal directions are perceived as properties of the visual field, functionally they originate in the observer. Configurational change, on the other hand, is entirely an environmental matter.) Rolling-wheel displays have been more recently investigated by Proffitt, Cutting, and Stier (1979). These authors consistently obtained vector analysis: the dots seemed to revolve about each other and simultaneously to undergo straight or wavy motions. We were interested in rolling-wheel displays because we wanted to see whether the findings of Wallach et al. (1985) applied also to motion patterns in which orientation change, rather than configurational change, operated. Would adding a stationary dot that could serve as a reference point for further orientation changes alter the results of orientation change? And would a motion process that resulted from orientation change sometimes combine with a subject-relative motion process to form a single motion path?

Because we feared it would be difficult to evaluate 
drawings of motion paths with cycloidal components, we used an arrangement with which Rubin (1927) experimented, in which a wheel rolled inside a hoop of twice its diameter. In this arrangement, the path of a point on the rim of the wheel is not a cycloid but is straight, and a combination of this straight subject-relative motion with the rotation caused by orientation change produces an easily described elliptic motion path.

The fact that, in Rubin's ingenious arrangement, cycloids become straight is easy to see. Because the circumference of the wheel is one-half of that of the inside of the hoop, a particular point on the rim of the wheel will touch the hoop twice during each full roll, at opposite ends of a diameter of the hoop.. That diameter will be the objective motion path of the point. The point will move repeatedly back and forth on this diameter in simple harmonic motion. Just as different rim points of a wheel rolling on a plane move on different cycloids, each rim point of Rubin's wheel will move on a different diameter of the hoop. If the diameter of the wheel is $2 r$, the length of the objective motion path of each rim point is $4 r$, for that is the diameter of the hoop.

When one rim point moves back and forth on its diameter of the hoop, it provides the corresponding subjectrelative stimulation, which causes a straight motion path to be perceived. If several rim points are visible and move on their diameters, they will reverse their motion directions at different times; this motion pattern may also be perceived as such. Alternatively, the rim points may form a group that undergoes an orientation change. In that case, they provide stimulation that results in the perception of a rotation of the group of points. When this happens, the group as a whole may have a motion of its own. This common motion, which, in our experiment, may or may not be seen, should have an elliptical path whose shape should depend on the distance between the points on the rim. When three points form the group, the common path, which is given as the subject-relative displacement of the group, is circular. ${ }^{1}$

\section{METHOD}

\section{Subjects}

Thirty-eight paid undergraduates, 19 men and 19 women, served as subjects.

\section{Equipment}

Instead of actually having a wheel roll inside a hoop, we used a different mechanism that meets the requirements of Rubin's motion arrangement. In Rubin's arrangement, the wheel rotates twice during a full revolution of its center, and, because it rolls, its rotation direction is opposite to the revolution of its center. Therefore, in our mechanism, a ring of light points rotated twice in one direction as the center of the ring revolved once about the center of the display in the other direction. Because the wheel fitted twice inside the hoop, the distance between the center of the wheel and the center of the display was equal to the radius, $r$, of the wheel, which was also the radius of the ring of lights. ${ }^{2}$

In our apparatus, $r$ was $8.5 \mathrm{~cm}$, and the length of the actual straight motion path of a light point was therefore $34 \mathrm{~cm}$. The light points were colored light spots, $6 \mathrm{~mm}$ across. Each consisted of a flashlight bulb over which a cover could be slipped; the cover was made of a plastic tube that ended in a plate with a circular opening that was covered with translucent material and a color filter. Six sockets for the bulbs were mounted on the display disk, $60^{\circ}$ apart on a circle of $8.5-\mathrm{cm}$ radius. The six light spots were therefore in positions that were the same as the points on the rim of the rolling wheel that our apparatus simulated. Because the light spots were $60^{\circ}$ apart, the straight paths on which they actually moved when the apparatus turned formed angles of $30^{\circ}$ with each other. ${ }^{3}$

In the present experiment, the apparatus was arranged so that the motion path of one of the light spots was vertical. We labeled the location of this light spot "I." Going in a clockwise direction about the circle, the next light location was called "II," and so on. The location of a moving light spot that coincided with the center of the circle on which Locations I to VI, the rim locations, were arranged was called "VII." Location VII moved on a circle, with a radius of $8.5 \mathrm{~cm}$, about the center of the whole display. There was also a location for a stationary light spot, $18 \mathrm{~cm}$ to the right of the display center, that is, $1 \mathrm{~cm}$ from the end of the path of the horizontally moving spot in Location IV.

\section{Procedure}

Two viewing distances were used, 5 and $10 \mathrm{~m}$. When the distance was $5 \mathrm{~m}$, the display subtended an angle of $3.9^{\circ}$, and when the subject fixated the stationary spot, the image of part of the display was outside the macula. This was avoided when the observation distance was $10 \mathrm{~m}$. A full rotation of the main shaft of the apparatus, which was also a full cycle of a rim spot's motion, took $2.5 \mathrm{sec}$.

There were six different displays, and each was observed under five viewing conditions. These 30 different presentations were given to each of our subjects in random order in two sessions of 15 presentations. The particular moving dots visible in the six displays were the following (an explanation of the italics is given in the description of Condition $\mathrm{C}$ below):

(1) Two rim dots, one vertical and one whose path formed an angle of $30^{\circ}$ with the vertical (Locations $I$ and VI).

(2) Two rim dots, one horizontal and one whose path formed an angle of $30^{\circ}$ with the horizontal (Locations $I V$ and III).

(3) Two rim dots, with each path forming an angle of $30^{\circ}$ with the vertical (Locations II and VI).

(4) Center of the circle and one rim dot $30^{\circ}$ from vertical (Locations VII and VI).

(5) Three rim dots, whose paths were $60^{\circ}$ apart, one of them horizontal and two forming an angle of $60^{\circ}$ with the horizontal (Locations IV, II, and $V I$ ).

(6) Three rim dots, one vertical, one $30^{\circ}$ from vertical and one $60^{\circ}$ from vertical (Locations I, VI, and III).

The five viewing conditions were the following:

(A) The moving dots were viewed freely. The subject was asked to scan the display constantly, looking quickly from one light dot to another. These instructions were meant to diminish the chance that the subject would track one of the light spots. Here the display was set in motion before it became visible to the subject.

(B) The instructions were the same as in Condition A, but the stationary dot was present and provided a landmark for the dot motions.

(C) The subject was instructed to track a particular moving dot, which was, in each case, identified by its color. Four covers for the bulbs of the displays, each with a different color filter, were available. The four different colors were assigned randomly to the two or three moving dots and to the stationary dot when it was visible. At each display, all subjects tracked the same dot. In the list of displays given above, the dot that was tracked and its location are italicized. In the tracking conditions, the display was stationary when it first became visible. Before the display was set in motion, the experimenter told the subject which dot to pursue.

(D) Instructions and procedures were the same as in Condition $\mathrm{C}$, but the stationary dot was visible.

(E) The stationary dot was visible and the subject was asked to 
fixate it during the exposure of the moving dots. Each display was stationary when it first became visible.

Two responses were obtained for each presentation. First, while the moving display was visible, the subject gave a verbal report of the motions of the spots. The instructions called for descriptions of the shapes and orientations of the two or three motion paths and the directions (senses) of the motions that went together. When the verbal report was complete, the display was covered. The subject turned on a nearby lamp and drew a picture showing the shapes and orientations of the two or three motion paths he or she had seen, while the experimenter recorded his or her verbal report. The subjects were informed beforehand that some dots might appear to undergo two motions simultaneously. A dot might, for instance, move on a circle, and the circular path might appear to move across at the same time. In that case, one motion was to be drawn as a solid line and the other as a dotted line. The purpose of the experiment was not known to the experimenter when she recorded the subjects' responses or when she later evaluated their drawings. ${ }^{4}$

For 18 subjects, 9 men and 9 women, the observation distance was $5 \mathrm{~m}$, and for 20,10 men and 10 women, it was $10 \mathrm{~m}$.

\section{RESULTS}

There are two conventional descriptions of the motions of points on the rim of Rubin's rolling wheel and, of course, of the dots in our displays. The dots either move back and forth on straight lines that pass through the center of the display and start their excursions at different times or they revolve about the center of a wheel which, in turn, revolves about the center of the display. The straight-line motion is given subject-relatively, and the revolving motion is given as a change in orientation when vector analysis occurs. Although perceived motion that resulted exclusively from subject-relative stimulation was always on a straight line, change in orientation did not always result in the dots' appearing to revolve about the wheel center. That occurred only in Displays 5 and 6, in which three well-spaced dots on the rim were visible. In displays with two rim dots, the given change in orientation was compatible with revolution of the dots about a point between them, and since, in Displays 1 and 2, the distance between the dots is $r$, the diameter of their revolutions may be as small as $r$ and not $2 r$, as it is when three dots on the rim revolve about a common center. ${ }^{5}$

But these were not the only motion patterns that our subjects reported. The motion of one of the dots might result from its participation in an orientation change and be circular, whereas the motion of the other dot might result from subject-relative stimulation and be linear. In addition, there were many reports of elliptic paths, combinations of circular and linear motions, the kind of motion paths whose occurrence we hoped to demonstrate. The shapes of the drawn ellipses varied widely. Very narrow ellipses, those with a width-to-length ratio of less than .1 , were counted as lines and are so listed in the tables. For statistical purposes, narrow and wide ellipses were distinguished. Those with width-to-length ratios between .1 and .3 were counted as narrow ellipses, and those with ratios larger than .3 as wide ellipses, with the former counted as more line-like.

Although the perception of common motions has no bearing on the issues of this paper, our subjects were asked to report them along with the motions of individual dots. Common motions were reported in viewing Conditions $\mathrm{A}$ and $\mathrm{B}$, which were conducive to their occurrence, in only half the instances in which all moving dots underwent circular motions. In that respect, the perceived motions that the Rubin arrangements yield resemble more the motions seen in some of Johansson's motion patterns than those caused when a wheel rolls on a plane. In connection with the 57 two-circle reports that were given for Displays 1, 2, and 3, common motions were reported 31 times; of the 46 three-circle reports that were given for the two three-rim-dot displays, only 23 were accompanied by common-motion reports. Also, the common motions that were reported were not always in agreement with expectation. For Displays 1 and 2 , in which the expected common motion paths were narrow ellipses, only straight motion paths were reported. For Display 1, there were 10 such nearly correct reports of vertical common motion out of a total of 11 reports, and for Display 2, there were 7 nearly correct reports of horizontal common motion out of a total of 9 reports. For Display 3, in which the expected path was a wider ellipse with the major axis vertical, 2 correct vertical oval paths were reported, in addition to 3 circular and 4 vertical linear paths, out of a total of 10 reports. In Displays 5 and 6, in which the expected common paths were circles, 14 circular paths were reported for a total of 23 common-motion reports. There were also 7 reports of linear motion and 2 reports that the common path seemed to change in shape. Apart from the results for Displays 1 and 2, only about half of the common paths reported agreed with expectation.

Table 1 lists 556 classifiable responses that were given to Displays 1,2, and 3, with two rim dots, during a total of 570 presentations. Table 2 lists 372 responses to a total of 380 presentations of Displays 5 and 6, with three rim dots. These responses are evaluated below, in connection with several issues.

\section{DISCUSSION}

\section{The Effect of Adding a Stationary Dot in the Periphery of the Display}

Would adding a stationary dot in the periphery of the moving-dot pattern have the same effect that adding stationary dots to the Johansson displays had in the experiments by Wallach et al. (1985)? There, introducing stationary reference points altered the pattern of configurational change in such a way that a large shift toward perception of the real motion paths took place. Presumably it had this effect by adding configurational changes that represented the real motion paths. Would the addition of the stationary dot in our experiment introduce further orientation changes and configurational changes that have a corresponding effect, namely, that of increasing the frequency with which linear motion is perceived and of causing alterations toward more line-like motion? There was another difference between the earlier experiments and the present one. Wallach et al. (1985) used several stationary dots, which caused the configurational change be- 
Table 1

Classification of Responses to Perceived Motion of Two Rim Dots in Displays 1, 2, and 3 Under Five Viewing Conditions of 114 Exposures Each

\begin{tabular}{|c|c|c|c|c|c|c|}
\hline $\begin{array}{c}\text { Two } \\
\text { Circles } \\
\frac{a}{a}\end{array}$ & $\begin{array}{l}\text { Circle, } \\
\text { Ellipse } \\
\mathrm{b}\end{array}$ & $\begin{array}{c}\text { Two } \\
\text { Ellipses } \\
\mathrm{c}\end{array}$ & $\begin{array}{l}\text { Line, } \\
\text { Circle } \\
d\end{array}$ & $\begin{array}{l}\text { Line, } \\
\text { Ellipse } \\
\mathrm{e}\end{array}$ & $\begin{array}{l}\text { Two } \\
\frac{\text { Lines }}{f}\end{array}$ & Total \\
\hline \multicolumn{7}{|c|}{ Free Viewing (A) } \\
\hline 42 & 10 & 14 & 27 & 9 & 6 & 108 \\
\hline \multicolumn{7}{|c|}{ Free Viewing with Stationary Dot (B) } \\
\hline 15 & 3 & 14 & 25 & 17 & 36 & 110 \\
\hline \multicolumn{7}{|c|}{ Pursuit (C) } \\
\hline 3 & 15 & 8 & 65 & 17 & 4 & 112 \\
\hline \multicolumn{7}{|c|}{ Pursuit with Stationary Dot (D) } \\
\hline 3 & 5 & 11 & 44 & 31 & 20 & 114 \\
\hline \multicolumn{7}{|c|}{ Fixation (E) } \\
\hline 11 & 1 & 23 & 12 & 17 & 48 & 112 \\
\hline Tot & & & & & & 556 \\
\hline
\end{tabular}

tween the moving dots to be overwhelmed by many added configurational changes, whereas here we used a single stationary dot, which participated in additional orientation and configurational changes and competed with the orientation change or changes among the moving dots.

A comparison of the results for the free-viewing Conditions $A$ and $B$ in Table 1 shows a change toward the perception of linear motion paths when, in Condition B, the stationary dot was added. The number of reports of two circles dropped from 42 to 15 , and two lines were seen 6 times in Condition A and 36 times in Condition B. The trend toward more line-like motion paths was subjected to a statistical test, which compared the motion reports given by individual subjects in Conditions $A$ and $B$, and then asked for the number of changes toward more line-like motion in Condition B and compared that number with the number of pairs of reports for the same displays that indicated an opposite change. There were 216 changes toward more line-like paths and 58 changes in the opposite direction. McNemar's test showed this difference to be highly significant $\left(\chi^{2}=91.11, p<.001\right)$. The results of Displays 4, 5, and 6 were included in this test.

Although this trend was strong in the case of displays with two rim dots (Table 1), it was just noticeable in displays with three rim dots (Table 2 ). In Table 1 , the totals of the circle-like reports, a, b, and c, were 66 in Condition $A$ and 32 in Condition $B$, and the totals of the more line-like reports, $d, e$, and f, were 42 and 78, respectively. In Table 2, the similar totals were 43 and 32 for the circlelike reports, a to d, and 33 and 44 for the line-like reports, e to $i$. More transparently put, the corrected sums, regardless of sign, of the differences of the two report categories, were $70 \times 2 / 3=47$ for the two-rim-dot displays and 22 for the three-rim-dot displays. We propose that the smaller effect of the stationary dot in the three-rim-dot displays is the outcome of a stronger group formation among the three moving dots. It strengthens the tendency toward circular motion, which is in competition with the orienta- tion changes introduced by the stationary dot. Support for this interpretation can be found in the results of the fixation conditions, $\mathrm{E}$, in which the line reports are the result of the dots' image paths, a potent subject-relative stimulation condition. For the displays with two rim dots listed in Table 1, there were 48 reports with two lines and 29 reports with one line, in contrast to 35 reports with circles or ellipses only. In other words, the ratio of more line-like reports to more circle-like reports was here 77 to 35 . The corresponding ratio for the results for the threedot displays (Table 2) was 31 to 45 . Here, too, the threedot displays yielded a higher rate of circle-like motions than did the two-dot displays.

\section{Limitation of Position Constancy}

Before we evaluate the elliptic path reports, we have to discuss the results of the pursuit conditions, C and D. Pursuing one of the dots causes its objective linear motion to become effective. Thus, it was perceived to move either linearly or, in combination with orientation change, on an elliptic path. Circle-circle reports were almost absent in Conditions C and D of Table 1. In Condition C, there was a preponderance of line-circle reports-65 out of a total of 107 reports. In Condition A, line-circle reports numbered only 27 . The increase by 38 to 65 cases resulted from the tracking of one of the dots, which changed many of the circle-circle reports in Condition A to line-circle reports. This explanation, however, implies that the circles that were seen when a line and a circle were reported resulted from orientation change, as it did when two circles were seen in Condition A. This assumption presupposes that position constancy operates in the perception of motion of one dot when the other dot is tracked. Becklen, Wallach, and Nitzberg (1984) found that position constancy does not operate while the eyes are engaged in pursuit movements if the target object has a motion of its own that is not colinear with the motion of the tracked object. This is just the case with the nontracked

Table 2

Classification of Responses to Perceived Motion of Three Rim Dots in Displays 5 and 6 Under Five Viewing Conditions of 76 Exposures for Each Condition

\begin{tabular}{|c|c|c|c|c|c|c|c|c|c|c|}
\hline & a & b & c & d & e & $\mathbf{f}$ & $\mathrm{g}$ & $\mathbf{h}$ & $\mathbf{i}$ & Total \\
\hline Number of Circles & 3 & 2 & 1 & - & - & 2 & - & 1 & - & \\
\hline Number of Ellipses & - & 1 & 2 & 3 & 2 & - & 1 & - & - & \\
\hline Number of Lines & - & - & - & - & 1 & 1 & 2 & 2 & 3 & \\
\hline \multicolumn{11}{|c|}{ Free Viewing (A) } \\
\hline & 28 & 8 & 0 & 7 & 6 & 12 & 2 & 1 & 12 & 76 \\
\hline \multicolumn{11}{|c|}{ Free Viewing with Stationary Dot (B) } \\
\hline & 18 & 6 & 0 & 8 & 7 & 17 & 5 & 1 & 14 & 76 \\
\hline \multicolumn{11}{|c|}{ Pursuit (C) } \\
\hline & 7 & 13 & 1 & 8 & 4 & 30 & 5 & $\mathbf{0}$ & 2 & 70 \\
\hline \multicolumn{11}{|c|}{ Pursuit with Stationary Dot (D) } \\
\hline & 7 & 8 & 3 & 7 & 4 & 24 & 12 & 3 & 6 & 74 \\
\hline \multicolumn{11}{|c|}{ Fixation (E) } \\
\hline & 10 & 3 & 1 & 5 & 10 & 16 & $\mathbf{0}$ & 2 & 29 & 76 \\
\hline Total & & & & & & & & & & 372 \\
\hline
\end{tabular}


dots in the pursuit condition of our experiments: in every instance, the tracked and the target dot move in different directions. In the absence of constancy, the perceived motion of the nontracked dot will depend primarily on the path of its retinal image, which is circular in all of our displays when a dot is tracked. ${ }^{6}$ This applies to 63 of the 65 listed line-circle reports; in two instances, the circular path was reported for the tracked dot. In the displays with three rim dots, tracking one of the dots in Condition $\mathrm{C}$ resulted in circular image paths for two dots. In Table 2, a toal of 30 line-two-circle reports are listed, in 27 of which both circle reports resulted from circular image paths.

\section{The Effect of Fixation}

Wallach et al. (1982) compared the effectiveness of ocular pursuit and of image path by putting each of these stimuli, representing subject-relative motion, in conflict with configurational change. A vertically moving dot was surrounded by a pattern of vertical lines in horizontal motion, which added horizontal induced motion to the objective vertical motion of the dot. The slope angle of the perceived motion path of the dot represented the result of the conflict between induced motion, that is, configurational change, on the one hand, and ocular pursuit, or image path, on the other. When induced motion was in conflict with ocular pursuit, it was fully effective, whereas when the dot's real motion was given by its image path, it yielded by about one-half . Our experiment, however, did not give corresponding results. There is no evidence that image paths are much more effective than ocular pursuit when they are in conflict with orientation change. In the two-rim-dot displays in Condition $\mathrm{E}$, in which a stationary dot was fixated, there were 48 two-line reports out of a total of 112 reports (lines were the image paths in this condition). This is, however, not a very high number, when it is considered in the context of the 36 twoline reports that occurred in Condition B, 30 of which were presumably caused by the mere presence of the stationary dot that was eventually fixated in Condition E. Orientation change may well be more potent than configurational change, ${ }^{7}$ and this may obscure the different effectivenesses of ocular pursuit and image path.

\section{Combination Paths}

We are now ready to deal with the other major purpose of our experiment-the demonstration that in the perception of our displays a motion process that results from subject-relative stimulation may combine with the process that results from orientation change. The combination of a straight motion that is given subject-relatively with a circular motion that results from orientation change is, as mentioned above, an elliptic path, of which our tables show many. Not all elliptic paths, however, resulted from such a process combination. Many ellipse reports that occurred in the pursuit conditions, $C$ and $D$, had nothing to do with orientation change. As already discussed, most of the circles seen in the pursuit conditions did not result from orientation change, but were caused by the circular image paths of the untracked dot. The same is true of the ellipses that were reported for the dots that were not tracked. Those ellipses did not result from the combination of straight-motion processes and of orientation change but were also caused by image paths, which were presumably elliptical because of inaccurate pursuit of the tracked straight motion. In the pursuit conditions, therefore, only the ellipses that were reported for the tracked dot's path resulted from process combination. Such ellipses occurred 39 times in Conditions $C$ and D of the two-rim-dot displays and 40 times in Conditions $C$ and $\mathrm{D}$ of the three-rim-dot displays. ${ }^{8}$

Although, in Condition C and D, the orientation change process combined with a subject-relative process that resulted from ocular pursuit, in the fixation condition, $\mathbf{E}$, the subject-relative process resulted from an image path, since here the objective straight dot motions were given in this manner. In condition $\mathrm{E}$ in Table 1 , there are, altogether, 41 reports of one or both dots that appeared to move on elliptic paths; in Table 2 there are 19 such reports. Since the ellipses resulted here from the combination of processes that were based on straight image paths and on orientation change, the ellipses contained in these 60 reports are cases in point.

Because eye movements during observation can cause straight image paths that result from the given straight dot motions to change into elliptic ones, which are then perceived, we do not know how many reported ellipses resulted from process combination in Conditions $A$ and $B$, where neither tracking nor fixation instructions were given. But the 79 ellipses that were reported in Conditions $\mathrm{C}$ and $\mathrm{D}$ as paths of pursued dots certainly resulted from process combination. Although we can be reasonably sure of the ellipses that occurred in the 60 reports of Condition $\mathrm{E}$, we preserve the integrity of this count by counting only wider ellipses, those with a width-to-height ratio of .3 or greater, and thus avoiding the inclusion of ellipses that might possibly have resulted from inadvertent eye movements. In the $\mathrm{E}$ conditions of all five displays, the total number of such wide ellipses was 44 . If the 79 cases from Conditions C and D are added, there are 123 assured instances of process combination among 558 classifiable responses.

\section{Summary}

Our experiment extended previous experimentation on vector analysis, in which configurational change operated as one of the stimulus conditions, to displays in which orientation change replaced configurational change. In the work by Wallach et al. (1985), apparent vector analysis was shown to depend on the simultaneous operation of two motion processes, one of which was produced by subject-relative stimulation and the other by configurational change, under circumstances in which one motion pattern provided the two stimulus conditions at the same time. Two results were obtained: (1) Adding stationary points in the surround of the motion pattern altered the 
pattern of perceived motions by introducing further configurational changes, and (2) it was shown that the two motion processes may combine and produce single paths that are different from either of the motion paths that the two stimulation conditions produced.

We obtained analogous results with a rolling-wheel display in which orientation change substituted for configurational change. Displays were used in which straight motions replaced the usual cycloidal paths of spots on the rim of the wheel. Hence, subject-relative stimulation represented straight motion, whereas orientation change represented the circular motion of the groups that the dots formed. Adding a stationary dot in the periphery of the displays diminished reports of circular motion and increased reports of straight motion, an effect analogous to the effects obtained by Wallach et al. (1985). The combination of the circular and the linear perceived motion was elliptical. Elliptical paths that resulted from such combinations occurred in our displays under two conditions, when the straight motions were given by ocular pursuit or as paths of retinal images.

Proffit et al. (1979) correctly state that "there are an indefinite number of different mathematical descriptions which would adequately describe the movement of ... stimulus lights" on a rolling wheel. They propose that "the perceptual system selects a particular description by placing a priority on the extraction of object-relative displacement"' (p. 302). We ascribe the selection of the perceived motions to the various conditions of stimulation that mediate motion perception and that represent different components of the objective motion.

Our results agree well with the observations that Duncker's (1929) subjects reported when they viewed two light points on a wheel that rolled on a plane. In Duncker's basic experiment (p. 241), one light point (C) was at the center of the wheel and another $(R)$ was on a radius halfway between the center and the rim. When the subjects were instructed to track point $R$, some saw it in circular motion; it either revolved about point $\mathrm{C}$ or both points were seen to revolve about their common center. Mostly, the two points together were here seen also in translatory horizontal motion, either straight or somewhat wavy. The former motions were caused by orientation change and the latter by subject-relative change. More frequent than these two versions was one in which point $R$ moved through a row of many loops, a path shaped like a drawing of a helical spiral. This path was obviously the result of a combination of the circular and the translatory motion process and corresponded to the elliptic path in our experiment. In this version, point $\mathrm{C}$ was seen in translatory motion, which was often wavy. When the subjects were instructed to track point $\mathrm{C}$, it always moved strongly up and down while undergoing its translation, another case of process combination. The motion of point $R$ was here mostly circular, in agreement with its image path, as Duncker correctly noted.

\section{REFERENCES}

Becklen, R., Wallach, H., \& Nitzberg, D. (1984). A limitation of position constancy. Journal of Experimental Psychology: Human Perception and Performance, 10, 713-723.

Duncker, K. (1929). Über induzierte Bewegung. Psychologische Forschung, 12, 180-259.

Johansson, G. (1950). Configurations in event perception. Uppsala: Almqvist \& Wiksells.

Proffit, D. R., Cutting, J. E. (1980). An invariant for wheelgenerated motions and the logic of its determination. Perception, 9 , 435-449.

Proffitt, D. R., Cutting, J. E., \& Stier, D. M. (1979). Perception of wheel-generated motions. Journal of Experimental Psychology: Human Perception and Performance, 5, 289-302.

RuBIN, E. (1927). Visuell wahrgenommene wirkliche Bewegungen. Zeitschrift für Psychologie, 103, 384-392.

WALLACH, H. (1965). Visual perception of motion. In G. Kepes (Ed.) The nature and an of motion. New York: Braziller.

WALLACH, H., BeCKLEN, R., \& NitZBERG, D. (1985). Vector analysis and process combination in motion perception. Journal of Experimental Psychology: Human Perception and Performance, 11, 93-102.

Wallach H., O'Leary, A., \& McMahon, M.L. (1982). Three stimuli for visual motion perception compared. Perception \& Psychophysics, 32, 1-6.

\section{NOTES}

1. This circular path of the group corresponds to the straight motion path that is observed when several points on the circumference of a wheel that rolls on a plane are visible. The elliptic path of two revolving rim points in our arrangement corresponds to the wavy path that is seen when two rim points are given that are not located on the same diameter of the wheel that rolls on a plane. For an explanation of the latter motions, see Proffitt and Cutting (1980).

2. The main shaft of our mechanism was located in the center of the display. It turned in the main bearing that was mounted on a sturdy support. The main shaft was oriented horizontally and coincided with the subject's line of sight. In the rear, the main shaft was connected to the slow shaft of a reduction-gear motor. A flat bar was attached at right angles to the front end of the main shaft. The bearing for the secondary shaft was inserted in this bar near its end. The display disk with the light points was attached to the front end of this secondary shaft. Therefore, when the main shaft turned, the display disk revolved around it. The distance between the centers of the two shafts was, of course, $r$.

The proper rotation of the disk about its center was obtained in the following fashion. A gear (A) was fastened on the secondary shaft in the rear of the bar. A second gear (B), whose diameter was twice that of gear A, was rigidly attached to the main bearing, concentrically with the main shaft, which was free to turn in the gear. Therefore, gear B was stationary and in the same plane as gear A. The two gears were connected by an idler gear that turned on a shaft that was inserted in the bar. When the main shaft turned and swung the bar around, the idler gear rolled on the stationary gear, B, and transmitted the rotation that resulted to gear $\mathbf{A}$. The light points on the display disk were arranged on a circle with the radius $r$ that was concentric with the secondary shaft.

3. One of us (H.W.) has used this apparatus to demonstrate in his lectures Rubin's original experiment. Rubin, who used the wheel-andhoop arrangement, had six evenly spaced light points on the rim of the wheel. Because they were all visible, Rubin always obtained vector analysis; his observers saw a pattern of six spots rotate about its center, and the whole pattern move in a circle. When H.W. had six light spots visible on his apparatus, his observers had the same experience.

4. In fact, the experiment was performed several years before the work of Wallach et al. (1985) was planned. It was originally meant to contribute to a comparison of the effects of orientation change and of configurational change. The context in which the results are here presented 


\section{WALLACH AND O'LEARY}

had not yet been thought of when the experiment was designed. This history of the experiment accounts for the presence of Display 4, whose results have no bearing on the issues discussed here and are not presented.

5. In their rolling-wheel experiments, Proffitt et al. (1979) also obtained revolutions with diameters smaller than that of the wheel when the distance between the light dots was smaller than the wheel diameter.

6. That the path of the retinal image of a dot is circular when another dot is tracked follows from the fact that the distance between the dots is fixed, since both are located on the same wheel.

7. The 63 line-circle reports in Condition $\mathrm{C}$, where the circles presumably resulted from image path, do not contradict this, because circular image paths do not conflict with orientation change, which also produces circular perceived paths.

8. Since the tables do not distinguish between elliptic paths of dots that were tracked and of dots that were not tracked, the number of process combination ellipses cannot be found in the tables.

(Manuscript received October 23, 1984; revision accepted for publication June 21,1985 .) 